\title{
Mitochondrial genome evolution in fire ants (Hymenoptera: Formicidae)
}

\author{
Dietrich Gotzek $^{1 *}$, Jessica Clarke ${ }^{2}$, DeWayne Shoemaker ${ }^{3}$
}

\begin{abstract}
Background: Complete mitochondrial genome sequences have become important tools for the study of genome architecture, phylogeny, and molecular evolution. Despite the rapid increase in available mitogenomes, the taxonomic sampling often poorly reflects phylogenetic diversity and is often also biased to represent deeper (family-level) evolutionary relationships.

Results: We present the first fully sequenced ant (Hymenoptera: Formicidae) mitochondrial genomes. We sampled four mitogenomes from three species of fire ants, genus Solenopsis, which represent various evolutionary depths. Overall, ant mitogenomes appear to be typical of hymenopteran mitogenomes, displaying a general A+T-bias. The Solenopsis mitogenomes are slightly more compact than other hymentoperan mitogenomes ( 15.5 kb), retaining all protein coding genes, ribosomal, and transfer RNAs. We also present evidence of recombination between the mitogenomes of the two conspecific Solenopsis mitogenomes. Finally, we discuss potential ways to improve the estimation of phylogenies using complete mitochondrial genome sequences.

Conclusions: The ant mitogenome presents an important addition to the continued efforts in studying hymenopteran mitogenome architecture, evolution, and phylogenetics. We provide further evidence that the sampling across many taxonomic levels (including conspecifics and congeners) is useful and important to gain detailed insights into mitogenome evolution. We also discuss ways that may help improve the use of mitogenomes in phylogenetic analyses by accounting for non-stationary and non-homogeneous evolution among branches.
\end{abstract}

\section{Background}

There has been a rapid proliferation of whole mitochondrial genomes (mitogenomes) sequenced in recent years, no doubt driven in part by the increasing speed and decreasing cost of sequencing technologies. Whole mitogenomes are increasingly used in phylogenetic studies [1-6] and in analyses of genome rearrangements [7-11], which can also be used for phylogenetic inference [8,12-16].

However, the utility of these datasets for these purposes greatly depends on taxon sampling. Currently, 237 insect mitogenomes have been fully sequenced (GenBank Sept. 21, 2009), yet the taxa utilized for these sequencing studies often do not reflect the distribution of species diversity. For example, Hymenoptera is one of

\footnotetext{
* Correspondence: gotzekd@si.edu

'Department of Ecology and Evolution, University of Lausanne, 1015

Lausanne, Switzerland

Full list of author information is available at the end of the article
}

the most species-rich insect orders $(\sim 130,000$ described species in 22 superfamilies [17]), yet only 11 mitogenomes have been fully sequenced (compared to 28 for beetles [300,000 species] and 69 for flies [110,000 species]). Despite a further seven hymenopteran mitogenomes being partially sequenced (Figure 1), taxon sampling still poorly reflects phylogenetic diversity of this important order across many taxonomic levels. Naturally, sampling is highly dependent on the questions a given researcher wishes to address with the data, yet biased sampling greatly limits the utility of the generated mitogenomic data in a comparative framework. For example, of the ten families of bees (Apoidea) and of the nine families of vespids (Vespoidea) only a single family (Apidae and Vespidae, respectively) of each superfamily has a sequenced mitogenome. But not only are many taxonomically and ecologically important families unsampled, there is also a dearth of mitogenomes for closely related species. We follow Gissi et al. [18] in arguing 


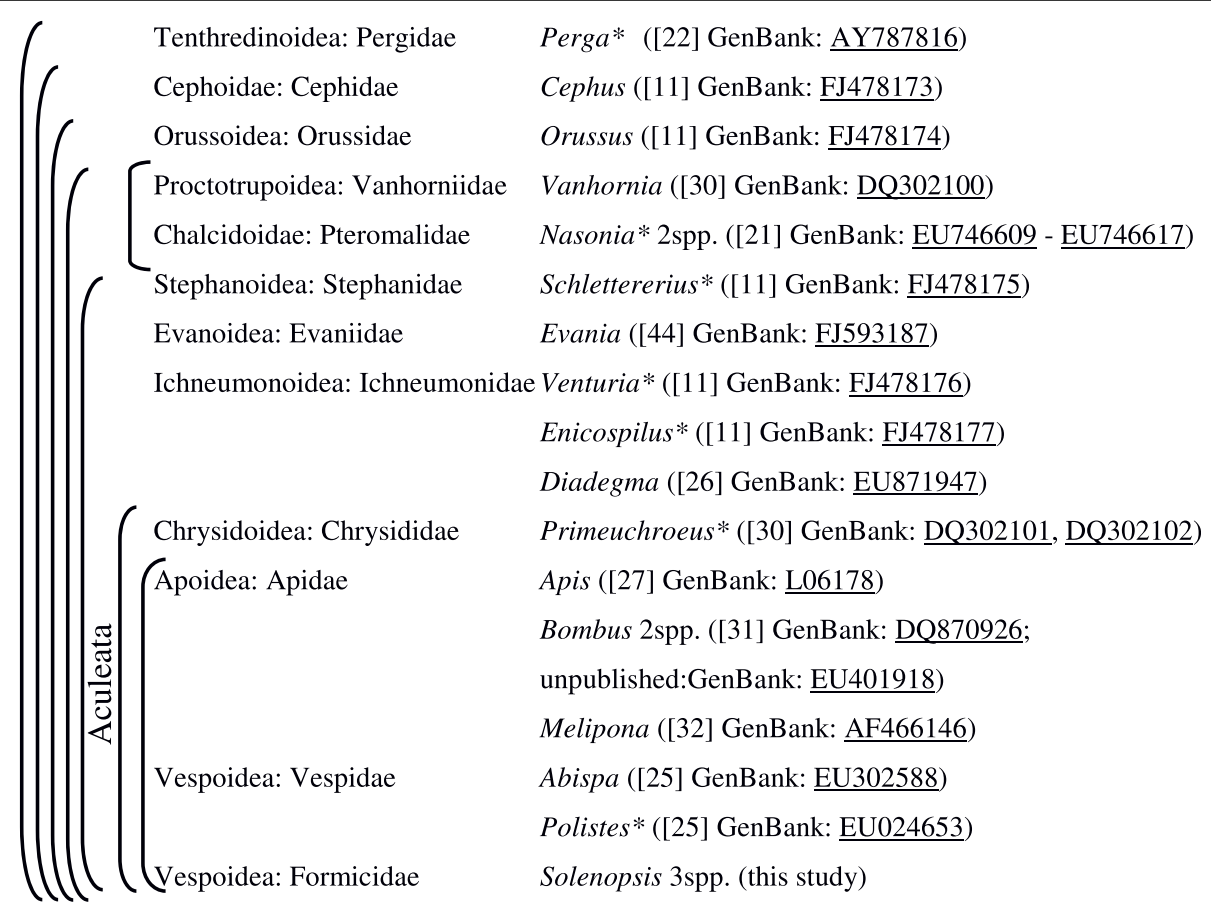

Figure 1 Available hymenopteran mitogenomes ordered phylogenetically (based on [11,54]). Parentheses indicate monophyly, superfamilies and families are assumed to be monophyletic. Asterices indicates partially sequenced genomes. Citations and GenBank accession numbers are also given.

that to better understand mitochondrial genome evolution we require an improved taxon sampling scheme that not only captures phylogenetic diversity more broadly but also takes into account various evolutionary depths, including variation within or among closely related species.

Hence, we here present the first complete mitogenomes of ants (Hymentopera: Formicidae; four mitogenomes from three fire ant species) and discuss their evolution in light of the other hymenopteran mitochondrial genomes. The four mitogenomes presented here represent important contributions to the ever expanding dataset of complete hymenopteran mitochondrial genomes in that they represent a previously unsampled, highly diverse, and ecologically dominant vespoid family (Formicidae). Our mitogenome data for three fire ant species belonging to two species groups $[19,20]$ also provides insights into mitogenome evolution at lower taxonomic levels. Such data generally are rare [18], with the notable exceptions in insects of a study employing comparable sampling for Nasonia [21] and much more extensive sampling in Drosophila (38 mitogenomes; GenBank Sept. 21, 2009).

\section{Results and Discussion}

For the present study we sequenced four complete mitogenomes from three fire ant species. These included two mitogenomes from Solenopsis invicta Buren, 1972 (referred to hereafter as "PMS" and "VMS" mitogenomes), one mitogenome from the closely related species S. richteri Buren, 1972 ("richteri" mitogenome) and one mitogenome from a more distantly related fire ant species S. geminata (Fabricius, 1804) ("geminata" mitogenome). These first complete mitogenome data for ants expand taxon selection of one of the largest and most diverse hymenopteran superfamilies (Vespoidea).

At first look, the mitogenomes of Solenopsis appear to be unremarkable compared with other hymenopteran mitogenomes, containing the same complement of protein-coding loci, tRNAs, rRNAs, and a pronounced A + T-bias (Table 1). Overall the ant mitogenomes are less divergent than those of Nasonia (3 - 13\% uncorrected nucleotide distance, $\pi$, compared with $\sim 15 \%$ among jewel wasps), however this comparison should be viewed with caution since it does not consider the potential differences in age among species within the Nasonia and Solenopsis species groups, which is not known. Also, we found no clear evidence of positive, directional selection acting on the ant mitogenomes $(\mathrm{dN} / \mathrm{dS} \approx 0.001-0.034$ for the 13 protein coding genes using the site model $[22,23]$ ).

\section{Gene content and order}

Consistent with other published hymenopteran mitogenomes, the four Solenopsis mitogenomes contain all 13 
Table 1 Summary of Solenopsis mitogenomes

\begin{tabular}{|c|c|c|c|c|c|c|c|c|}
\hline & $\begin{array}{l}\text { length } \\
\text { (bp) }\end{array}$ & PCG+rRNA & tRNAs & $\begin{array}{l}\text { overall } \\
\text { AT bias }\end{array}$ & $\begin{array}{l}\text { protein } \\
\text { AT bias }\end{array}$ & ENC & CBI & $\begin{array}{c}\text { scaled } \\
\chi^{2}\end{array}$ \\
\hline VMS & 15,548 & $13+2$ & 22 & 77.5 & 74.5 & 42.971 & 0.563 & 0.103 \\
\hline PMS & 15,549 & $13+2$ & 22 & 77.2 & 74.2 & 43.270 & 0.543 & 0.106 \\
\hline richteri & 15,560 & $13+2$ & 22 & 76.9 & 74.0 & 43.477 & 0.534 & 0.099 \\
\hline geminata & 15,552 & $13+2$ & 22 & 76.5 & 73.5 & 44.695 & 0.524 & 0.126 \\
\hline
\end{tabular}

PCG: protein coding genes; ENC: effective number of codons (out of a maximum of 61) [92]; CBI: Codon Bias Index [91]. The scaled $\chi^{2}$ [93] used to test for codon bias was corrected for $\mathrm{G}+\mathrm{C}$ bias using Yates' correction.

protein-coding genes and both rRNAs in the same order and direction of the hypothesized ancestral pancrustacean mitogenome (Figure 2). As in the three Nasonia species [21], the four Solenopsis mitogenomes share identical architectures at the tRNA loci as well. However, the three methods we used to identify tRNAs greatly differed in sensitivity and accuracy (Table 2). Both DOGMA (used at COVE cut-off score $=20$ ) and ARWEN over-predicted tRNAs (usually well over 30 for DOGMA and up to 26 by ARWEN). As a result, they usually identified more of the 22 tRNAs than tRNAscan-SE. tRNAscan-SE was more conservative, although some predictions were not well supported (COVE scores $<20)$. It also misidentified tRNAs three times: two tRNA-F in geminata (COVE $=19.47)$ and at the same position in VMS $(\mathrm{COVE}=18.65)$ and tRNA-P $(\mathrm{COVE}=$ 23.85) also in VMS. Since all of these tRNAs overlap with SrRNA (tRNA-F) or nad5 (tRNA-P) and have relatively low COVE scores, we considered them false positives. Only twice were none of the methods able to detect tRNAs: tRNA- $S_{1}$ in geminata and tRNA-N in VMS. However, we were able to manually fold these tRNAs.

The location of tRNAs differs from the hypothetical ancestral hymenopteran mitogenome [24], which is also a typical feature of hymenopteran mitogenome architecture $[11,25]$. However, with only three apparent translocations, the Solenopsis mitogenome architecture appears to be less derived than that of the other hymenopteran mitogenomes [11,25-32]. Most mitogenome rearrangements in
Hymenoptera appear to be selectively neutral and involve tRNA translocations around the coxII - atp 8 junction $[29,30]$ and the $n d 3-n d 5$ junction $[11,25]$. The Solenopsis mitogenomes show no variation in the coxII - atp 8 junction and a translocation of tRNA-N from the $n d 3-n d 5$ junction to the SrRNA - nd2 junction. The tRNA-V translocation also moved to the SrRNA - nd2 junction. Various types of gene order rearrangements are generally recognized, differing by their location (local vs. distant) and whether they retain their original orientation (inverted vs. not inverted) and generally can be classified into one of several categories: local inversions, local translocations (gene shuffling), translocations, and remote inversions (an inverted translocation) [25].

Visual inspection of the source locations for these two translocations led us to test the manner of the rearrangement, since short sequences of the length of typical tRNAs (approximately 60-80 bp) remained at the source locations (hereafter termed "degenerate" tRNAs). A duplication/loss model of translocation seems plausible for the tRNA-V translocation, whereas this model seems less likely for the tRNA-N rearrangement, since this rearrangement also involves an inversion (remote inversion). We consider an intra-mitochondrial recombination event [28] an unlikely mechanism in this particular case as well, since the translocation spanned half of the mitogenome and none of the intermediate genes are inverted or rearranged. Since DOGMA and ARWEN had placed an additional tRNA-N in the same position but opposite orientation as the tRNA-D in the VMS

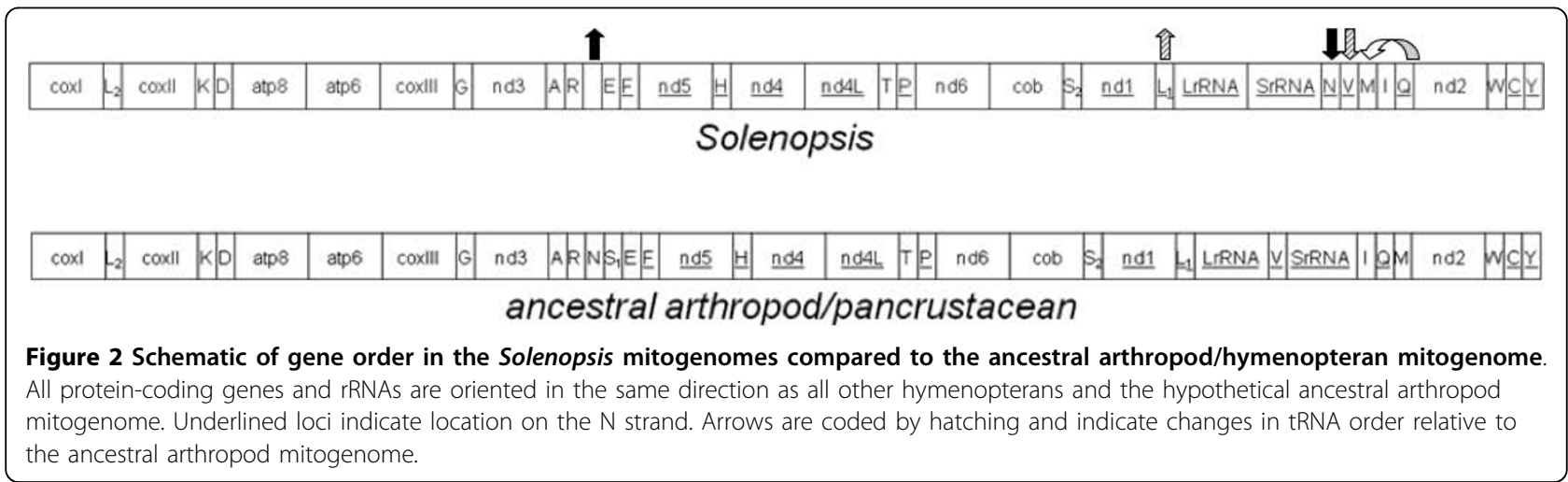


Table 2 Comparison of various methods to identify tRNAs in the Solenopsis mitogenomes

\begin{tabular}{|c|c|c|c|c|c|c|c|c|c|c|c|c|c|}
\hline \multirow[b]{2}{*}{$\begin{array}{l}\text { tRNA } \\
\text { Type }\end{array}$} & \multirow[b]{2}{*}{$\begin{array}{l}\text { Anti } \\
\text { Codon }\end{array}$} & \multicolumn{3}{|c|}{ geminata } & \multicolumn{3}{|c|}{ richteri } & \multicolumn{3}{|c|}{ PMS } & \multicolumn{3}{|c|}{ VMS } \\
\hline & & $\begin{array}{c}\text { DOGMA } \\
\text { Cove } \\
\text { score }\end{array}$ & $\begin{array}{c}\text { tRNAscan } \\
\text { Cove } \\
\text { Score }\end{array}$ & ARWEN & $\begin{array}{c}\text { DOGMA } \\
\text { Cove } \\
\text { score }\end{array}$ & $\begin{array}{c}\text { tRNAscan } \\
\text { Cove } \\
\text { Score }\end{array}$ & ARWEN & $\begin{array}{c}\text { DOGMA } \\
\text { Cove } \\
\text { score }\end{array}$ & $\begin{array}{c}\text { tRNAscan } \\
\text { Cove } \\
\text { Score }\end{array}$ & ARWEN & $\begin{array}{c}\text { DOGMA } \\
\text { Cove } \\
\text { score }\end{array}$ & $\begin{array}{c}\text { tRNAscan } \\
\text { Cove } \\
\text { Score }\end{array}$ & ARWEN \\
\hline $\mathrm{L}_{2}$ & TAA & 47.63 & 22.63 & yes & 49.15 & 31.79 & yes & 49.15 & 31.79 & yes & 49.15 & 31.06 & yes \\
\hline K & $\pi T$ & 44.74 & 18.29 & yes & 39.21 & 21.78 & yes & 39.21 & 21.78 & yes & 37.64 & 21.78 & yes \\
\hline $\mathrm{D}$ & GTC & 47.83 & 24.42 & yes & 41.13 & 18.89 & yes & 42.77 & 18.09 & yes & 42.79 & 18.9 & yes \\
\hline G & TCC & 58.63 & 36.52 & yes & 49.5 & 32.45 & yes & 51.34 & 29.97 & yes & 48.23 & 29 & yes \\
\hline $\bar{A}$ & TGC & 46.06 & 19.45 & yes & 48.9 & 24.57 & yes & 48.9 & 24.57 & yes & 54.71 & 25.98 & yes \\
\hline $\mathrm{R}$ & TCG & 45.47 & 19.34 & yes & 39.41 & - & yes & 39.41 & - & yes & 38.96 & - & yes \\
\hline$\overline{S_{1}}$ & $\mathrm{TCT}$ & - & - & - & 26.43 & - & yes & 26.43 & - & yes & 27.89 & - & - \\
\hline$\underline{E}$ & $\pi \mathrm{TC}$ & 53.66 & 25.17 & yes & 52.91 & 24.39 & yes & 52.91 & 24.39 & yes & 52.91 & 24.39 & yes \\
\hline F & GAA & 46.74 & 30.61 & yes & 38.05 & 24.63 & yes & 38.49 & 28.14 & yes & 38.26 & - & yes \\
\hline$\underline{\mathrm{H}}$ & GTG & 54.21 & 20.57 & yes & 43.51 & 18.42 & yes & 43.51 & 18.42 & yes & 43.51 & 18.42 & yes \\
\hline $\bar{T}$ & TGT & 58.18 & 27.74 & yes & 53.59 & 29.42 & yes & 53.59 & 29.42 & yes & 58.58 & 24.55 & yes \\
\hline$\underline{P}$ & TGG & 51.68 & 26.94 & yes & 56.84 & 22.42 & yes & 56.84 & 22.42 & yes & 59.46 & 25.1 & yes \\
\hline$S_{2}$ & TGA & 48.14 & 20.98 & yes & 48.14 & 20.98 & yes & 48.14 & 20.98 & yes & 48.14 & 20.98 & yes \\
\hline$\underline{\underline{L}}_{1}$ & TAG & 25.07 & 18.66 & yes & 19.23 & 17.8 & - & 20.18 & 18.97 & yes & - & 16.85 & yes \\
\hline $\mathrm{N}$ & ATT & 25.55 & 15.34 & - & 32.4 & - & - & 32.4 & - & yes & - & - & - \\
\hline$\underline{\underline{V}}$ & TAC & 48.34 & 18.03 & yes & 59.55 & 33.94 & yes & 59.55 & 33.94 & yes & 55.58 & 31.31 & yes \\
\hline $\bar{M}$ & CAT & 42.75 & 18.84 & yes & 44.28 & 15.68 & yes & 43.92 & - & yes & 42.53 & 20.11 & yes \\
\hline 1 & GAT & 36.35 & - & yes & 36.59 & 15.13 & yes & 36.18 & 19.8 & yes & 37.02 & 21.35 & yes \\
\hline$\underline{\mathrm{Q}}$ & TTG & 53.61 & 42.39 & yes & 51.22 & 41.64 & yes & 51.22 & 41.64 & yes & 53.25 & 42.31 & yes \\
\hline W & TCA & 52.16 & 27.57 & yes & 47.34 & 30.06 & yes & 48.69 & 27.06 & yes & 44.83 & 26.98 & yes \\
\hline$\underline{c}$ & GCA & 56.88 & 28.08 & yes & 61.16 & 27.44 & yes & 57.83 & 26.43 & yes & 57.44 & 28.93 & yes \\
\hline$\underline{Y}$ & GTA & 44.94 & 20.78 & yes & 46.2 & 26.86 & yes & 43.75 & 24.44 & yes & 47 & 24.81 & yes \\
\hline
\end{tabular}

DOGMA and ARWEN generally overpredicted tRNAs (data not shown) and tRNAscan underpredicted tRNA loci. COVE scores $>20$ are generally considered to indicate reliable identification of tRNAs. Some tRNAs were readily identified by all methods, whereas some proved harder to identify and had to be manually folded in some mitogenomes (e.g., tRNA-S $)_{1}$. Loci underlined are located on the $\mathrm{N}$ strand.

mitogenome (data not shown), we included all hymenopteran tRNA-N, - V, and -D loci in a phylogenetic analysis with the relevant Solenopsis tRNAs. The resulting tree placed the tRNA-D, tRNA-V, and degenerate
tRNA-N firmly among their respective tRNA species, whereas the degenerate tRNA-V and new tRNA-N are clearly not closely related to any other tRNAs (Figure 3 ). This suggests that the identification of the overlapping

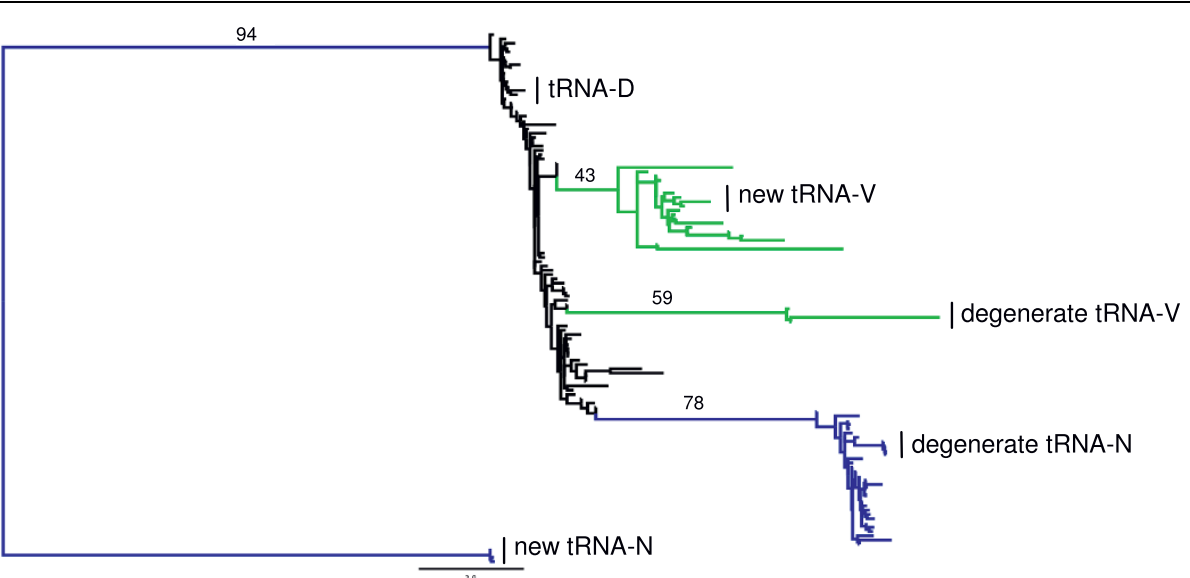

Figure 3 Maximum likelihood phylogeny of hymenopteran tRNAs. Only the Solenopsis tRNAs are labeled. Black branches identify tRNA-D loci. Blue identify all tRNA-N loci, including "new" and "degenerated" loci in Solenopsis. Green branches are tRNA-V loci, again with the "new" and "degenerated" loci labeled. The values above the long major branches are bootstrap support (100 replicates). 
tRNA-N over tRNA-D in VMS was erroneous. Also, the "new" translocated tRNA-V is clearly homologous to the other hymenopteran tRNA-V genes, suggesting a real translocation event. However, the identity of the degenerate locus is less clear. Since it forms a distinct and highly supported clade, we are inclined to interpret this as a duplication/loss translocation and the "degenerate" tRNA-V as a vestigial spacer, which we initially erroneously hypothesized to be a degenerate tRNA due to its position and length. Finally, the source of the tRNA$\mathrm{N}$ is not clear and at present since we are unable to determine the source tRNA in our dataset (data not shown), but it is becoming increasingly clear that our understanding of tRNA evolution is rapidly changing [33-43]. We will require better sampling of other mitogenomes to shed light on the evolution of this tRNA.

Visual inspection of tRNAs across species reveals that there is considerable amount of variation due to point mutations and indels in almost all tRNAs. This variation results in differences in ability of the three tRNA identification methods to correctly identify tRNAs across species (Table 2), suggesting that congeneric comparative studies of tRNAs are fruitful endeavors to studying tRNA evolution and mitgenome architecture [18].

\section{Nucleotide and codon bias}

The four Solenopsis mitogenomes are consistently A+Tbiased, but this bias is less pronounced than in any of the other apocritan Hymenoptera ( 1-10\% less; Figure 4A). Wei et al. [44] describe the same pattern for a distantly related evanid wasp, which suggests that the $\mathrm{A}+\mathrm{T}$-bias is perhaps more variable across the hymentoperan phylogeny than previously realized. There was no significant difference in nucleotide bias of the four ant mitogenomes (Table 1). Additionally, a T-bias persisted across protein coding regions on the coding strand (Figure 5). This was especially pronounced across the second codon positions. No evidence for codon bias was found after correcting for nucleotide bias (Table 1).

\section{Recombination}

Recombination in animal mitogenomes has been well documented [45-47]. Nonetheless, such recombination remains difficult to detect reliably, no doubt in part due to the lack of power of many analytical methods developed for detecting recombination [48]. This pattern is reflected in our data set as well, as most methods were unable to detect statistically significant signatures of recombination. However, three local methods found significant support for a single recombination event within the PMS mitogenome (Table 3, Figure 6), with an approximately 500 bp surrounding the $n d 6$ - cytb junction (nucleotide positions $8,971-9,517$ of the aligned
Solenopsis mitogenomes) derived from a VMS-like ancestor.

Consistent with this scenario, six additional methods of analysis showed statistically non-significant yet congruent signals of recombination in the same location. One likely reason for the comparatively weak support for the hypothesized recombination event is that the translocation could have occurred sufficiently long ago that the signal of the event has decayed. Our results are unlikely an experimental artifact, since the recombinant sequence was recovered from separate PCR (and sequencing) reactions in which the initial PCR reactions utilized different conserved primers flanking this region. Further, the sequence data were high quality, the sequences differ slightly from the other three sequences, and the sequence traces do not show any evidence of conflicting base calls.

\section{Phylogeny}

The phylogenetic tree recovered from all four Solenopsis mitogenomes is identical to a tree generated previously using sequence data from a much shorter region (coxI/ tRNA- $\mathrm{L}_{2} /$ coxII) and demonstrates that the S. invicta mitogenomes are not monophyletic: the PMS invicta mitogenome is more closely related to richteri than the VMS invicta mitogenome [49] (Figure 4). This phylogenetic pattern is upheld throughout the complete mitochondrial alignment, except for the short fragment shown above to have been transferred during the recombination event (Figure 6).

Our phylogenetic analysis of all hymenopteran mitogenomes encountered similar problems described previously [6,50-52] in that some expected relationships were not recovered, e.g. the sister taxa relationship between the Proctotrupomorpha (Nasonia and Vanhornia; sensu Rasnitsyn [53]) and the remainder of the Apocrita $[50,54]$ or the sister relationship of the Ichneumonoidea (represented by Ichneumonidae in this study) with the Aculeata (Vespomorpha sensu Rasnitsyn [53]) $[6,51,52,54]$. Also, our phylogenetic analyses were unable to support the monophyly of the Vespoidea (ants and vespids; Figure 4) due to the placement of Solenopsis outside of the Vespidae + Apoidea clade with high branch support. While ants generally are considered to belong to the Vespoidea [55], some doubts have been raised regarding the monophyly of this superfamily $[54,56]$ and our results mirror those of Dowton et al. [52] using only the mitochondrial $16 \mathrm{~S}$ rRNA locus.

Our homotachous (site specific rates do not change along tree) Bayesian analysis recovered a topology fully concordant with that of Dowton et al. [6] (Figure 4B). This was not surprising, since we tried to follow the suggestions made by Dowton et al. [6] regarding the best analysis parameters for hymentoperan mitogenomic 
A.

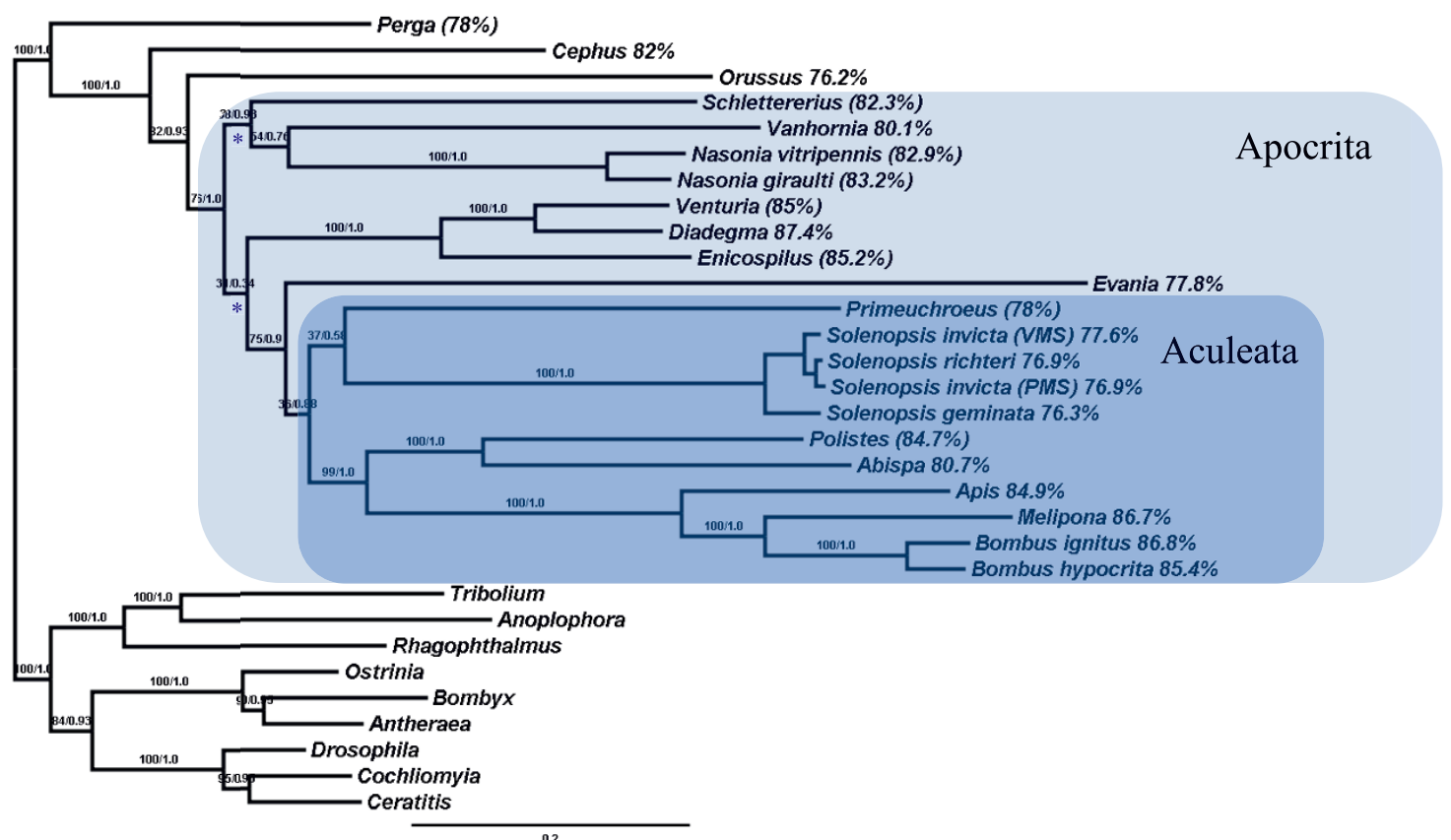

B.

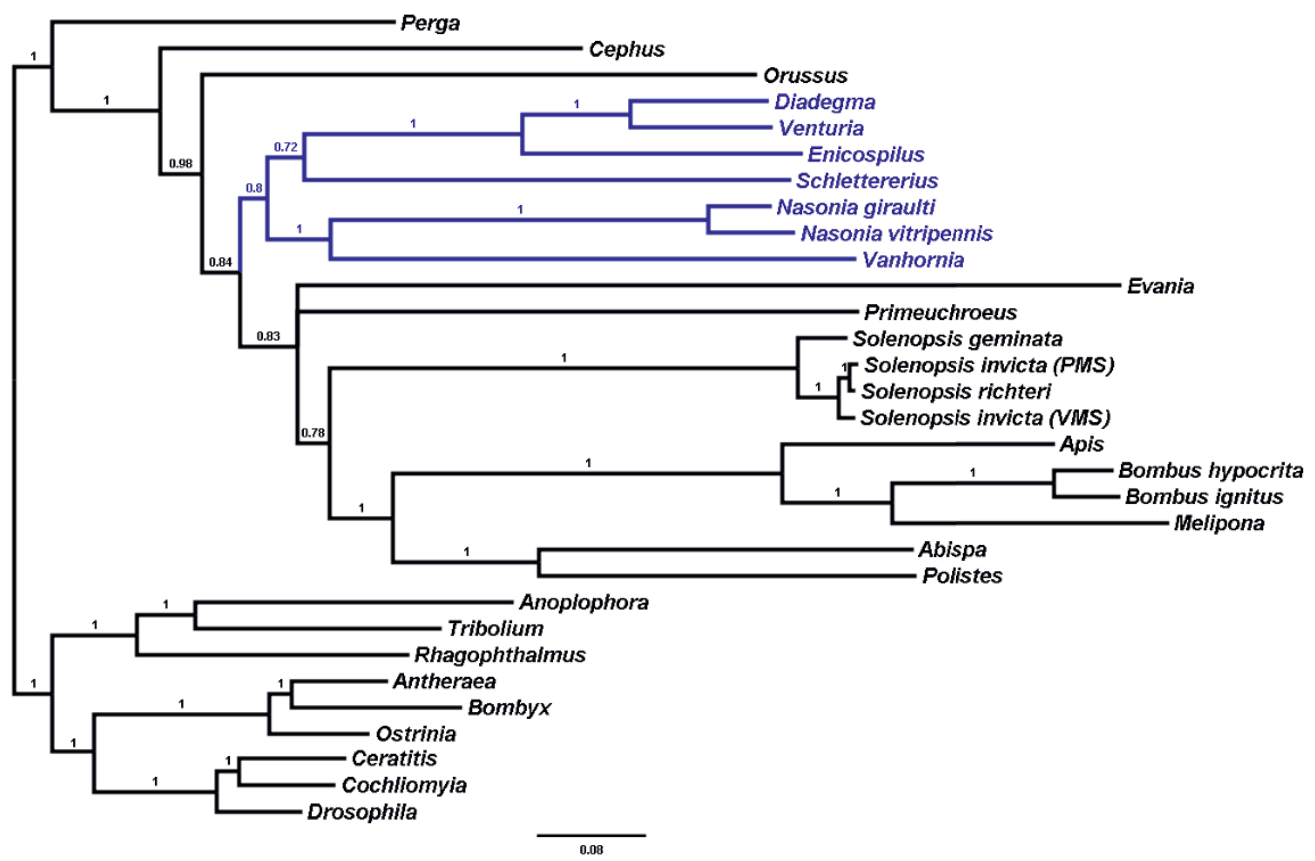

Figure 4 Phylogenetic hypotheses of Hymenoptera based on protein coding genes and rRNA genes. A. Phylogeny recovered from maximum likelihood analyses. Topology of the homogeneous (PhyML) and non-homogeneous (nhPhyML) analyses were identical. Values above internal branches are bootstrap (100 replicates) and aLRT (SH-like) branch support estimates. Values for percent A+T-bias are shown after genus name (values in parentheses are calculated from incomplete genome sequences). The apocritan and aculeatan clades are highlighted. The blue stars indicate posterior probabilities of 0.85 in the heterotachous Bayesian analysis (see B below); the other branches in this part of the tree were recovered with posterior probabilities of 1.0. B. Phylogeny derived from Bayesian inference. The tree topology between the homogenous and heterotachous analyses are identical, except for the clade highlighted in blue: the heterotachous analysis recovered phylogenetic relationships of these taxa which are identical to the maximum likelihood analyses shown in A. Bayesian posterior probabilities are given for the homogeneous model of nucleotide substitution, which is identical to that of the heterotachous model except for the clade in blue (see A above). 


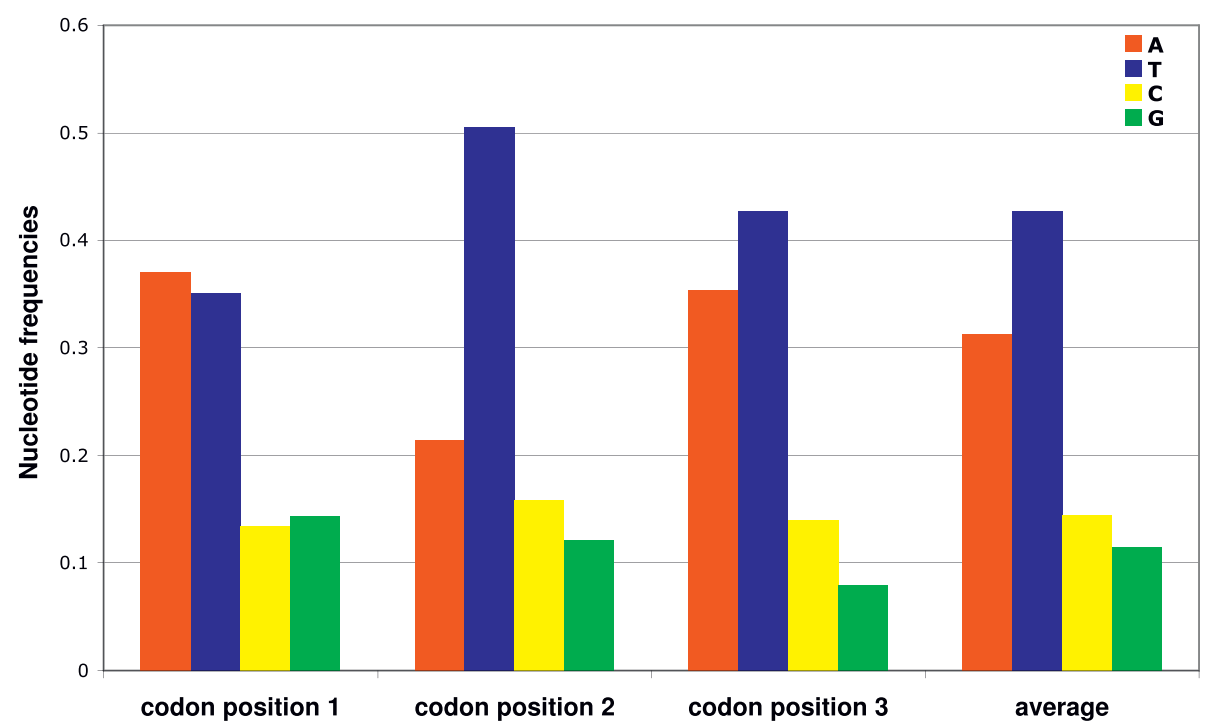

Figure 5 Average nucleotide bias in protein coding genes averaged across all 4 Solenopsis mitogenomes. There are no significant differences between mitogenomes (data not shown).

data (i.e., using nucleotide data, exclusion of $3^{\text {rd }}$ codon positions, sampling several outgroups, and using a partitioned Bayesian framework). This suggests that the phylogenetic inference was robust to additional taxon sampling (Solenopsis, Diadegma, Evania). However, this topology differed from our other analyses in the placement of Ichneumonidae. Whereas the homotachous

Table 3 Results of tests of recombination for the translocation of 500 bp fragment at nt8,950-9,483.

\begin{tabular}{ccccc}
\hline Program & Test & Probability & Type of test & Ref. \\
\hline RDP3 & RDP & $\mathbf{0 . 0 0 8 7}$ & local & {$[103]$} \\
& GENECONV & 0.6320 & local & {$[104]$} \\
& BootScan & $\mathbf{0 . 0 2 1 2}$ & local & {$[105]$} \\
& MaxChi & 0.3103 & local & {$[106]$} \\
& Chimaera & 0.8262 & local & {$[107]$} \\
& Sister Scan & 0.6969 & local & {$[108]$} \\
& 3Seq & 0.3514 & - & {$[109]$} \\
& Distance plot & yes & local & \\
& PhylPro & yes & - & {$[110]$} \\
& LARD * & - & - & {$[111]$} \\
TOPALi2.5 & DSS & no & local & {$[112]$} \\
& LRT & YES & local & \\
& PDM & no & - & {$[113]$} \\
& HMM & no & - & {$[114]$} \\
RecombiTest & GENECONV* & 0.0929 & global & {$[115]$} \\
& MaxChi *i & 0.85 & global & {$[106]$} \\
\hline
\end{tabular}

Significant probability values are indicated in bold, statistically insignificant values that still show a pattern of recombination at that position are italicized (only applies to local methods). Asterices (*) mark methods used to verify recombination detected by other methods. ${ }^{1}$ was run on $5.5 \mathrm{~kb}$ window around the $500 \mathrm{bp}$ recombinant.
Bayesian analysis recovered the Ichneumonidae as a sister group to Schlettererius (Stephanidae), the other analyses placed the Ichneumonidae basal to an Aculeata + Evania clade. The analyses also differed in the placement of Primeuchroeus. Both maximum likelihood methods placed Primeuchroeus with Solenopsis, whereas both Bayesian analyses left its position unresolved at the base of the other Aculeata. However, all of these placements were poorly supported regardless of the branch support measure. Our analyses suggest that optimality criteria and models of nucleotide evolution have a stronger influence on the resulting phylogenetic inference in our dataset than taxon sampling. The importance of optimality criterion was previously also noted by Dowton et al. [6] and suggests that the extraction of phylogenetic signal from hymenopteran mitogenomic data is not simple.

While Dowton et al. [6] showed that the accuracy of mitogenomic phylogenetic analysis is greatly improved by the implementation of a particular partitioning scheme in a Bayesian framework, their analyses failed to account for heterotachous (site specific rate of evolution changes along the tree) and non-stationarity (base frequencies change along the tree) substitution processes between branches during phylogenetic analyses [57-60], of which the latter is an especially well-known feature of the hymenopteran mitogenomes $[[6,44,61,62]$ this study]. Hence, we compared the phylogenies derived under homotachous and stationary models of nucleotide substitution with those allowing site specific rates of change and base frequencies to change between branches. We implemented the covarion model [59] in 


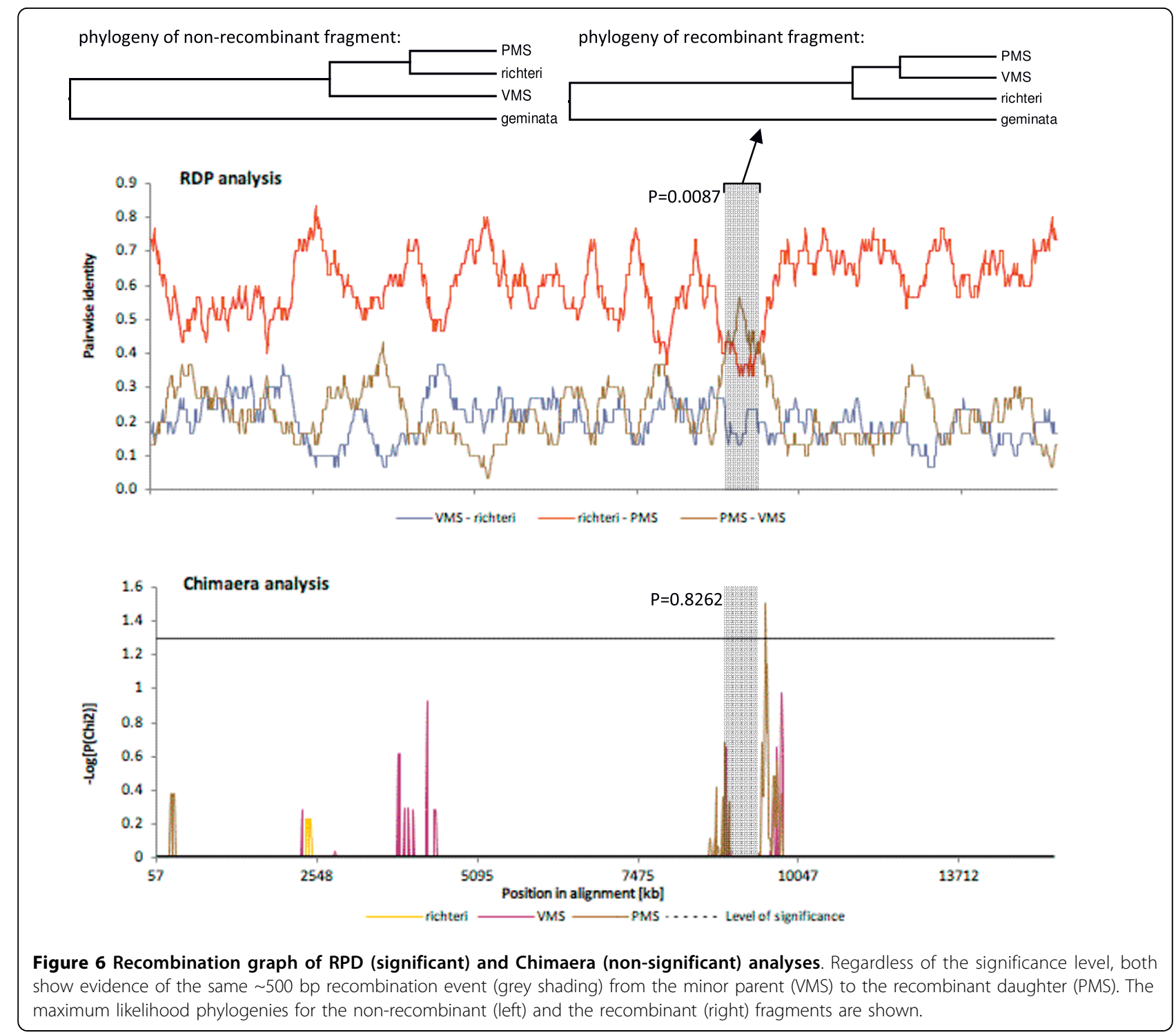

MrBayes to accommodate heterotachy and the model of Galtier and Gouy [57] to account for non-stationarity (and non-homogeneity) using nhPhyML. Applying Galtier and Gouy's [57] model under the maximum likelihood criterion significantly improved the fit of the model to the data (nhPhyML: $-\operatorname{lnL}=159,198.79453$; PhyML: $-\operatorname{lnL}=160,821.701853 ; \mathrm{df}=60$, $\mathrm{LRT}=$ $3245.8125, \mathrm{p}<0.0001$ [58]), but it did not result in a different topology (Figure 4A). Use of the covarion model (which accommodates heterotachy by allowing sites to change their substitution rate along branches by being switched on or off) in the Bayesian framework did not result in an improvement in log-likelihood (covarion model: $-\ln \mathrm{L}=156,475.84$; stationary model: $-\ln \mathrm{L}=$ 156,228.88), but recovered a different topology almost identical to that recovered under the maximum likelihood optimality criterion (Figure 4B). This decrease in log-likelihood of the covarion model was surprising [but see [60]], but the exact implementation of heterotachy may be important $[60,63]$ so the evaluation of other heterotachous models (e.g., mixed branch length model [64] or the RERV model [65]) may be warranted. More thorough studies are undoubtedly required to tease apart the contribution and possible interaction between different models and optimality criteria $[4,6,60,63,66]$.

It is evident that reconstructing the hymenopteran phylogeny using only mitogenome data is problematic due to a variety of factors, e.g, differences in GC-content and rates of evolution among branches [64] or an ancient rapid diversification event $[67,68]$ which is known to greatly complicate phylogenetic inference [69]. To account for these confounding factors, one should 
attempt to break up long branches by increased taxon sampling wherever possible. Including several species per genus (Nasonia, Bombus, and Solenopsis) as we have done is not very effective. Hence we suggest adding more genera and previously unsampled higher level taxa to more effectively break up long branches. While increased taxon sampling will likely rapidly occur in the near future, we agree with earlier suggestions of the importance of not relying on mitogenomes as the sole source of data for inferring phylogenetic relationships [70]. Mitochondria essentially provide one very specific evolutionary history of a lineage since they are maternally inherited as single markers independently from the nuclear genome, and their utility can be further compromised if their transmission is influenced by intracellular symbionts such as Wolbachia [70]. Given the rapidly decreasing costs in high-through-put sequencing and the various rapidly increasing genomic resources for several apocritan lineages (Apis and Nasonia genomes [71-73], Solenopsis, Camponotus, Vespula, and Microctonus genomic libraries [74-78]), we are hopeful that future phylogenetic work will be based on a broader genetic basis employing many more molecular characters [72].

\section{Conclusions}

To fully clarify the evolution of mitochondrial genomes in the Hymenoptera will require much greater sampling at all taxonomic levels (i.e., from the superfamily to the species and possibly intraspecific level), which should provide much needed data to fully address the patterns and rate of evolution and genome organization in this organelle. While this information will yield an increased understanding of hymenopteran evolutionary history, mitogenome studies need to be complemented by phylogenetic studies utilizing other sources of data against which we can compare and contrast the information yielded by whole mitochondrial genome analyses. Given the recent rate of publications with full mitogenomes, this will no doubt occur in the near future, yet could be greatly optimized through concerted sampling efforts by the greater scientific community.

\section{Methods}

\section{Generation of sequence data}

Complete mitochondrial genomes were generated for four individuals from three closely related Solenopsis species. The two $S$. invicta samples were collected in Pascagoula, MS (PMS) and Vera, MS (VMS), S. geminata was collected in Tallahassee, FL, and S. richteri was collected in Rosario, Argentina. Even though the two S. invicta mitogenomes were sequenced from specimens collected in the invasive range, the $\sim 1 \mathrm{~kb}$ coxI/ tRNA- $\mathrm{L}_{2} /$ coxII section of these mitogenomes are identical to haplotypes from the native range (PMS is H22 [GenBank: AY249114]; VMS is H5 [GenBank: AY249097]) and represent two widely divergent clades within S. invicta and two geographically and genetically distinct populations (Argentina and Brazil) [49,79]. We sequenced the entire mitogenome of each individual using a primer walking approach by performing 34-40 separate PCRs of genomic portions of the genome of variable size ( 400-1,000 bp). We designed primers such that substantial overlap occurs between the various amplicons (allowing independent verification of sequence data by increasing depth of coverage) and such that the combination of all PCR amplicons spanned the entire genome. We initially attempted to amplify portions of the genome using conserved primers published in Simon et al. [80] as well as by designing conserved primers for a subset of coding genes by aligning available mitogenome sequences for Apis mellifera [GenBank: NC001566] and Drosophila yakuba [GenBank: NC001322]. We subsequently designed additional primers spanning other regions with no or low coverage using sequence data generated for fire ants. All primers developed and used for this study are presented in Additional file 1.

Several lines of evidence suggest that our sequences generated specifically represent mitochondrial genomic DNA rather than nuclear mitochondrial-like sequences (numts), which appear to be common and are generally short and highly fragmented in ants and other Hymenoptera [81-83]: The redundant yet independent PCR amplification of essentially every genomic region, the absence of ambiguous base calls characteristic of heterozygotes, the PCR amplification of the complete genome, and the generation of a contig identical in sequence to PMS using 454 pyrosequencing technology (DDS unpublished data).

All PCR amplicons were sequenced in both directions and each strand was assembled into single contigs with overlapping ends, indicating that our mitogenome sequences contained no gaps. Leading and lagging strand for each mitogenome were then aligned and manually checked for indels or ambiguous base calls. Mitogenomes were deposited in the NCBI GenBank database [GenBank: HQ215537, HQ215538, HQ215539, HQ215540].

\section{Genome annotation}

Mitogenomes were annotated using the DOGMA webserver [84], which uses BLASTX against a custom database to identify protein coding genes. We verified all annotations made with DOGMA: coding regions were checked against a S. invicta EST database [75] and tRNAs were validated using ARWEN 1.2 [85] and tRNAscan-SE 1.21 [86] since DOGMA only uses COVE 
[87] to identify tRNAs. Generally, tRNAscan-SE has very low false positive rates and thus rarely mispredicts tRNAs (COVE scores $\geq 20$ are usually considered reliable [86]), whereas ARWEN has a low false negative rate and usually identifies all tRNAs [85]. Generally, DOGMA identified significantly more tRNAs than either ARWEN or tRNAscan-SE, sometimes with quite high COVE scores. Two tRNAs in particular were not recovered, tRNA-S $S_{1}$ and tRNA-N. These, however could be folded manually.

\section{Sequence analyses}

Nucleotide sequences were aligned based on amino acid alignments using MUSCLE 3.6 [88]. Models of nucleotide evolution were estimated for protein coding genes using jModeltest [89]. DnaSP 4.50.3 [90] was used to estimate codon usage bias and nucleotide frequency bias [91-93]. The CODEML program in the PAML4.2 package [94] was used to test for site-specific evidence of positive selection while correcting for nucleotide bias [95]. We employed the following parameters: runmode $=0$, omega and kappa estimated (from three different starting points), empirical codon frequencies from each codon position (codonfreq $=2$ ).

Following the recommendations of Posada [48], we employed a suite of recombination detection programs offered in the program packages TOPALi 2.5 [96] and RPD 3b32 [97] and the RecombiTest website [46]http:// www.lifesci.sussex.ac.uk/CSE/test/index.php to test for recombination in the Solenopsis mitogenomes (see Table 3 for specific tests used). When any of the recombination tests only utilized three sequences at a time (e. g., RDP), analyses were repeated with every possible sequence triplet combination and p-values were Bonferroni corrected. All settings were left at the software default for the initial analyses, except for the PDM and LRT, where we used flexible window sizes. The highest acceptable $p$-value was 0.05 (unless Bonferroni corrected). Loosely following Tsaousis et al.'s [45] criteria for evidence of recombination, we consider as good evidence for recombination when more than one test detected a recombination event (although without regard to the test being a global or a local method). The more tests recovering evidence for recombination the more confident we are that it represents a true recombination event. Although this classification is admittedly arbitrary, we agree with White et al. [98] that identifying instances of recombination is inherently difficult and requires the heuristic use of several methods to identify potential recombinants.

Phylogenetic analyses were conducted on protein coding genes of the hymentoperan mitogenomes and 9 outgroups (3 flies [GenBank: X03240, AF260826, AJ242872], 3 beetles [GenBank: AJ312413, DQ768215,
AB267275], and 3 moths [GenBank: AF442957, AF149768, AY242996]). jModeltest [89] was used to estimate the most appropriate model of nucleotide evolution for each codon position at each locus separately. Following the suggestion of Dowton et al. [6] we used the Bayesian approach using nucleotide sequences and implemented the GTR $+\mathrm{I}+\Gamma$ model of sequence evolution across genes and codon positions since jModeltest usually indentified this model as the best fitting for each data partition. MrBayes 3.1.2 [99] was then used to recover phylogenetic hypotheses. All parameters were unlinked between partitions. Two independent analyses were run for three million generations, each with three heated and one cold chain. Parameters were sampled every $1000^{\text {th }}$ generation. Convergence between runs was assessed when log-likelihoods had plateaued, PRSF factors were $\sim 1$, and split frequencies had dropped $<0.01$. Samples taken prior to convergence were removed before samples were summarized. The same analysis was repeated implementing the covariotide model of sequence evolution to account for heterotachy (changes in site-specific evolutionary rates across lineages) [59], which has been shown to effectively accommodate heterotachy [[66], but see [60]]. Since this analysis took longer to converge, 5 million generations were run.

Maximum likelihood analyses were implemented on the PhyML 3.0 webserver [100] http://www.atgc-montpellier.fr/phyml/. We implemented the GTR $+\mathrm{I}+\Gamma$ model of nucleotide substitution on the unpartitioned dataset, estimated proportion of invariable sites and gamma shape parameter using six substitution rate categories, and optimized equilibrium frequencies, branch lengths, and tree topology (using the nearest-neighbor interchange [NNI] and sub-tree pruning and regrafting [SPR]) on five random starting trees. In addition to running one hundred bootstrap replicates to estimate levels of branch support, we also implemented the SH-like aLRT, which assesses the likelihood gain of the presence of that branch [101]. To accommodate non-stationarity (changes of base frequencies between branches) we implemented nhPhyML-Discrete [58] using default options and the topology recovered from the heterotachous Bayesian analysis as the starting tree.

The evolution of tRNA-N was studied using phylogenetic analyses as suggested by Saks et al. [34] and Dowton and Austin [29], which were conducted using 100 bootstrap replicates in PhyML using the same configuration as described above. Other relevant hymenopteran tRNAs (D, N, and V) were downloaded from GenBank and aligned using MUSCLE. Unlike other authors $[29,37,38]$, unpaired loops and anticodons were not removed following the suggestions of Wong et al. [102]. However, we would like to point out that the phylogenetic analysis should only be interpreted as a heuristic 
tool, since the alignment of many very short, evolutionary very old, and highly AT-biased sequences is not trivial, regardless of alignment method used or prior editing to remove problematic areas.

\section{Additional material}

\section{Additional file 1: Primers used for PCR amplification and} sequencing of fire ant genomes. Primers names and sequences used in this study. $J$ and $\mathrm{N}$ within primer names refer to heavy and light strands, respectively, and indicate the orientation of the primers. Primer names beginning with "Gem" were designed specifically to amplify the mitogenome of $S$. geminata.

\section{Acknowledgements}

We would like to thank Kenneth Ross (UGA) and Seán Brady (NMNH, Smithsonian Institution) for critically reviewing the manuscript. We also thank four anonymous reviewers for their comments which greatly improved the manuscript. This study was supported in part by a grant from the United States Department of Agriculture NRICGP (2006-35302-16561; Agreement No. 58-6615-7-137).

\section{Author details}

'Department of Ecology and Evolution, University of Lausanne, 1015 Lausanne, Switzerland. 'Department of Entomology, University of Wisconsin, Madison, Wisconsin, USA. ${ }^{3}$ USDA-ARS Center for Medical, Agricultural, and Veterinary Entomology, 1600/1700 SW 23rd Drive, Gainesville, Florida, USA.

\section{Authors' contributions}

JC and DDS carried out all aspects of the molecular lab work. DG and DDS performed all analyses of the data and wrote the manuscript. All authors read and approved the final manuscript.

Received: 29 October 2009 Accepted: 7 October 2010 Published: 7 October 2010

\section{References}

1. Arnason U, Adegoke JA, Gullberg A, Harley EH, Janke A, Kullberg M: Mitogenomic relationships of placental mammals and molecular estimates of their divergences. Gene 2008, 421:37-51.

2. Dong S, Kumazawa Y: Complete mitochondrial DNA sequences of six snakes: phylogenetic relationships and molecular evolution of genomic features. J Mol Evol 2005, 61:12-22.

3. Cameron SL, Lambkin CL, Barker SC, Whiting MF: A mitochondrial genome phylogeny of Diptera: whole genome sequence data accurately resolve relationships over broad timescales with high precision. Syst Entomol 2007, 32:40-59.

4. Sheffield NC, Song H, Cameron SL, Whiting MF: Nonstationary evolution and compositional heterogeneity in beetle mitochondrial phylogenomics. Syst Biol 2009, 58:381-394.

5. Fenn JD, Song H, Cameron SL, Whiting MF: A preliminary mitochondrial genome phylogeny of Orthoptera (Insecta) and approaches to maximizing phylogenetic signal found within mitochondrial genome data. Mol Phylogenet Evol 2008, 49:59-68.

6. Dowton M, Cameron SL, Austin AD, Whiting MF: Phylogenetic approaches for the analysis of mitochondrial genome sequence data in the Hymenoptera - A lineage with both rapidly and slowly evolving mitochondrial sequences. Mol Phyl Evol 2009, 52:512-519.

7. Boore $\mathrm{J}$, Lavrov DV, Brown WM: Gene translocation links insects and crustaceans. Nature 1998, 392:667-668.

8. Dowton M: Relationships among the cyclostomebraconid (Hymenoptera: Braconidae) subfamilies inferred from a mitochondrial tRNA gene rearrangement. Mol Phylogenet Evol 1999, 11:283-287.

9. Cameron SL, Johnson KP, Whiting MF: The mitochondrial genome of the screamer louse Bothriometopus (Phthiraptera: Ischnocera): effects of extensive gene rearrangements on the evolution of the genome. $J \mathrm{Mol}$ Evol 2007, 65:589-604.

10. Beckenbach AT, Joy JB: Evolution of the mitochondrial genomes of gall midge (Diptera: Cecidomyiidae): Rearrangements and several truncation of the tRNA genes. Genome Biol Evol 2009, 1:278-287.

11. Dowton M, Cameron SL, Dowavic Jl, Austin AD, Whiting MJ: Characterization of 67 mitochondrial tRNA gene rearrangements in the Hymenoptera suggests that mitochondrial tRNA gene position is selectively neutral. Mol Biol Evol 2009, 26:1607-1617.

12. Boore JL, Brown WM: Big trees from little genomes: mitochondrial gene order as a phylogenetic tool. Curr Opin Genet Dev 1998, 8:668-674.

13. Smith MJ, Arndt A, Gorski S, Fajber E: The phylogeny of echinoderm classes based on mitochondrial gene arrangements. J Mol Evol 1993, 36:545-54.

14. Boore JL, Collins TM, Stanton D, Daehler LL, Brown WM: Deducing the pattern of arthropod phylogeny from mitochondrial DNA rearrangements. Nature 1995, 376:163-165.

15. Kumazawa $Y$, Nishida M: Variations in mitochondrial tRNA gene organization of reptiles as phylogenetic markers. Mol Biol Evol 1995, 12:759-772.

16. Curole JP, Kocher TD: Mitogenomics: digging deeper with complete mitochondrial genomes. Trends Ecol Evol 1999, 14:394-398.

17. Grissell EE: Hymenopteran biodiversity: some alien notions. Am Entomol 1999, 45:235-244.

18. Gissi $C$, lannelli F, Pesole G: Evolution of the mitochondrial genome of Metazoa as exemplified by the comparison of congeneric species. Heredity 2008, 101:301-320.

19. Trager JC: A revision of the fire ants, Solenopsis geminata group (Hymenoptera: Formicidae: Myrmicinae). J New York Entomol Soc 1991, 99:141-198.

20. Pitts J, McHugh Ross KG: Cladistic analysis of the fire ants of the Solenopsis saevissima species-group (Hymenoptera: Formicidae). Zool Scripta 2005, 34:493-505.

21. Oliveira DC, Raychoudhury R, Lavrov DV, Werren JH: Rapidly evolving mitochondrial genome and directional selection in mitochondrial genes in the parasitic wasp Nasonia (Hymenoptera: Pteromalidae). Mol Biol Evol 2008, 25:2167-2180.

22. Nielsen R, Yang Z: Likelihood models for detecting positively selected amino acid sites and applications to the HIV-1 envelope gene. Genetics 1998, 148:929-936

23. Yang Z, Nielsen R, Goldman N, Pedersen A-MK: Codon-substitution models for heterogeneous selection pressure at amino acid sites. Genetics 2000, 155:341-449.

24. Castro LR, Dowton M: The position of the Hymenoptera within the Holometabola as inferred from the mitochondrial genome of Perga condei (Hymeoptera: Symphyta: Pergidae). Mol Phyl Evol 2005, 14:469-479.

25. Cameron SL, Dowton M, Castro LR, Ruberu K, Whiting MF, Austin AD, Diement K, Stevens J: Mitochondrial genome organization and phylogeny of two vespid wasps. Genome 2008, 51:800-808.

26. Wei S-J, Shi M, He J-H, Sharkey M, Chen X-X: The complete mitochondrial genome of Diadegma semiclausum (Hymenoptera: Ichneumonidae) indicates extensive independent evolutionary events. Genome 2009 52:308-319.

27. Crozier RH, Crozier YC: The mitochondrial genome of the honeybee Apis mellifera - complete sequence and genome organization. Genetics 1993, 133:97-117.

28. Dowton M, Castro LR, Campbell SL, Bargon SD, Austin AD: Frequent mitochondrial gene rearrangements at the hymenopteran nad3-nad5 junction. J Mol Evol 2003, 56:517-526.

29. Dowton M, Austin AD: Evolutionary dynamics of a mitochondrial rearrangement "hot spot" in the Hymenoptera. Mol Biol Evol 1999, 16:298-309.

30. Castro LR, Ruberu K, Dowton M: Mitochondrial genomes of Vanhornia eucnemidarum (Apocrita: Vanhorniidae) and Primeuchroeus spp. (Aculeata: Chrysididae): evidence of rearranged mitochondrial genomes within the Apocrita (Insecta: Hymenoptera). Genome 2006, 49:752-766.

31. Cha SY, Yoon HJ, Lee EM, Yoon MH, Hwang JS, Jin BR, Han YS, Kim I: The complete nucleotide sequence and gene organization of the mitochondrial genome of the bumblebee, Bombus ignitus (Hymenoptera: Apidae). Gene 2007, 392:206-220. 
32. Silvestre $D$, Dowton $M$, Arias MC: The mitochondrial genome of the stingless bee Melipona bicolor (Hymenoptera, Apidae, Meliponini): Sequence, gene organization and a unique tRNA translocation event conserved across the tribe Meliponini. Genet Mol Biol 2008, 31:451-460

33. Cantatore P, Gadaleta MN, Roberti M, Saccne C, Wilson AC: Duplication and remoulding of tRNA genes during the evolutionary rearrangement of mitochondrial genomes. Nature 1987, 329:853-855.

34. Giegé R, Sissler M, Florentz $C$ : Universal rules and idiosyncratic features in tRNA identity. Nucl Acid Res 1998, 26:5017-5035.

35. Saks ME, Sampson JR, Abelson J: Evolution of a transfer RNA gene through a point mutation in the anticodon. Science 1998, 279:1665-1670.

36. Tocchini-Valentini G, Saks ME, Abelson J: tRNA Leucine identity and recognition sets. J Mol Biol 2000, 298:779-793.

37. Higgs $P G$, Jameson $D$, Jow $H$, Rattray $M$ : The evolution of tRNA-Leu genes in animal mitochondrial genomes. J Mol Evol 2003, 57:435-445.

38. Rawlings TA, Collins TM, Bieler R: Changing identities: tRNA duplication and remolding within animal mitochondrial genomes. Proc Nat'l Acad Sci USA 2003, 100:15700-15705.

39. Xue H, Tong KL, Marck C, Grosjean H, Wong JT: Transfer RNA paralogs: evidence for genetic code-amino acid biosynthesis coevolution and an archeal root of life. Gene 2003, 310:59-66.

40. Lavrov DV, Lang BF: Transfer RNA recruitment in mitochondrial DNA. Trends Genet 2005, 21:129-133.

41. Tang DTP, Glazov EA, MCWilliam SM, Barris WC: Dalrymple: Analysis of the complement and molecular evolution of tRNA genes in cow. BMC Genomics 2009, 10:188

42. Rogers HH, Bergman CM, Griffiths-Jones S: The evolution of tRNA genes in Drosophila. Genome Biol Evol 2010, 2:467-477.

43. Seligmann $\mathrm{H}$ : Undetected antisense tRNAs in mitochondrial genomes? Biology Direct 2010, 5:39.

44. Wei S-J, Tang $P$, Zheng L-H, Shi M, Chen X-X: The complete mitochondrial genome of Evania appendigaster (Hymenoptera: Evaniidae) has low A+T content and a long intergenic spacer between atp8 and atp6. Mol Biol Rep 2010, 37:1931-1942.

45. Tsaousis AD, Martin DP, Ladoukakis ED, Posada D, Zouros E: Widespread recombination in published animal mtDNA sequences. Mol Biol Evol 2005, 22:925-933.

46. Piganeau $G$, Gardner M, Eyre-Walker A: A broad survey of recombination in animal mitochondria. Mol Biol Evol 2004, 21:2319-2325.

47. Ujvari B, M Dowton, T. Madsen: Mitochondrial DNA recombination in a free-ranging Australian lizard. Biol Lett 2007, 3:189-192.

48. Posada D: Evaluation of methods for detecting recombination from DNA sequences: empirical data. Mol Biol Evol 2002, 19:708-717.

49. Shoemaker DD, Ahrens ME, Ross KG: Molecular phylogeny of fire ants of the Solenopsis saevissima species-group based on mtDNA sequences. Mol Phylogenet Evol 2006, 38:200-215.

50. Castro LR, Dowton M: Molecular analyses of the Apocrita (Insecta: Hymenoptera) suggests that the Chalcidoidea are sister to the diaprioid complex. Invert Syst 2006, 20:603-614.

51. Dowton M, Austin AD: Molecular phylogeny of the insect order Hymenoptera: Apocritan relationships. Proc Natl Acad Sci USA 1994, 91:9911-9915.

52. Dowton M, Austin AD, Dillon N: Bartowsky: Molecular phylogeny of the apocritan wasps: the Proctotrupomorpha and Evaniamorpha. Syst Entomol 1997, 22:245-255.

53. Rasnitsyn AP: An outline of evolution of the hymenopterous insects (Order Vespida). Oriental Insects 1988, 22:115-145.

54. Sharkey MJ: Phylogeny and classification of Hymenoptera. Zootaxa 2007, 1668:521-548.

55. Brothers DJ: Phylogeny and evolution of wasps, ants and bees (Hymenoptera, Chrysidoidea, Vespoidea, and Apoidea). Zool Scripta 1999, 28:233-249.

56. Pilgrim EM, von Dohlen CD, Pitts JP: Molecular phylogenetics of Vespoidea indicate paraphyly of the superfamily and novel relationships of its component families and subfamilies. Zool Scripta 2008, 37:539-560.

57. Galtier N, Gouy M: Inferring pattern and process: maximum-likelihood implementation of a nonhomogeneous model of DNA sequence evolution for phylogenetic analysis. Mol Biol Evol 1998, 15:871-879

58. Boussau B, Gouy M: Efficient likelihood computations with non-reversible models of evolution. Syst Biol 2006, 55:756-768.
59. Huelsenbeck JP: Testing a covariotide model of DNA substitution. Mol Biol Evol 2002, 19:698-707.

60. Kolaczkowski B, Thornton JW: A mixed branch length model of heterotachy improves phylogenetic accuracy. Mol Biol Evol 2008, 25:1054-1066.

61. Castro $L R$, Austin $A D$, Dowton $M$ : Contrasting rates of mitochondrial molecular evolution in parasitic Diptera and Hymenoptera. Mol Biol Evol 2002, 19:1100-1113.

62. Dowton M, Austin AD: Increased genetic diversity in mitochondrial genes is correlated with the evolution of parasitism in the Hymenoptera. J Mol Evol 1995, 41:958-965.

63. Wang $\mathrm{H}-\mathrm{C}$, Specer $\mathrm{M}$, Susko E, Roger AJ: Testing for covarion-like evolution in protein sequences. Mol Biol Evol 2006, 24:294-305.

64. Kolaczkowski B, Thornton JW: Performance of maximum parsimony and likelihood phylogenetics when evolution is heterogeneous. Nature 2004, 431:980-984

65. Wu J, Susko E, Roger AJ: An independent heterotachy model and its implications for phylogeny and divergence time estimation. Mol Phyl Evol 2008, 46:801-806

66. Zhou Y, Rodrigue N, Lartillot N, Philippe $\mathrm{H}$ : Evaluation of the model handling heterotachy in phylogenetic inference. BMC Evol Biol 2007 7:206.

67. Grimaldi D, Engel MS: Evolution of the Insects Cambridge: Cambridge University Press 2005

68. Brady SG, Larkin L, Danforth BN: Bees, ants, and stinging wasps (Aculeata). In The Timetree of Life. Edited by: Hedges SB, Kumar S. Oxford: Oxford University Press: 2009:264-269.

69. Whitfield JB, Kjer KM: Ancient rapid radiations of insects: Challenges for phylogenetic analysis. Ann Rev Entomol 2008, 53:449-472.

70. Hurst GDD, Jiggins FM: Problems with mitochondrial DNA as a marker in population, phylogeographic and phylogenetic studies: the effects of inherited symbionts. Proc R Soc B 2005, 272:1525-1534.

71. Honeybee Genome Sequencing Consortium: Insights into social insects from the genome of the honeybee Apis mellifera. Nature 2006, 443:931-49.

72. Savard J, Tautz D, Richards S, Weinstock GM, Gibbs RA, Werren JH, Tettelin H, Lercher MJ: Phylogenomic analysis reveals bees and wasps (Hymenoptera) at the base of the radiation of Holometabolous insects. Genome Res 2006, 16:1334-1338.

73. Nasonia Genome Working Group: Functional and Evolutionary Insights from the Genomes of Three Parasitoid Nasonia Species. Science 2010, 327:343-348

74. Wilfert L, Muñoz-Torres M, Reber-Funk C, Schmid-Hempel R, Tomkins Gadau J, Schmid-Hempel P: Construction and characterization of a BAClibrary for a key pollinator, the bumblebee Bombus terrestris L. Insect Soc 2009, 56:44 - 48.

75. Wang J, Jemielity S, Uva P, Wurm Y, Gräff J, Keller L: An annotated cDNA library and microarray for large-scale gene-expression studies in the ant, Solenopsis invicta. Genome Biol 2007, 8:R9.

76. Goodisman MAD, Isoe J, Wheeler DE, Wells MA: Evolution of insect metamorphosis: A microarray-based study of larval and adult gene expression in the ant Camponotus festinatus. Evolution 2005, 59:858-870.

77. Hoffman EA, Goodisman MAD: Gene expression and the evolution of phenotypic diversity in social wasps. BMC Biology 2007, 5:23.

78. Crawford AM, Brauning R, Smolenski G, Ferguson C, Barton D, Wheeler TT, McCulloch A: The constituents of Microctonus sp. parasitoid venoms. Insect Mol Biol 2008, 17:313-324.

79. Ross KG, Krieger MJB, Keller L, Shoemaker DD: Genetic variation and structure in native populations of the fire ant Solenopsis invicta: evolutionary and demographic implications. Biol J Linn Soc 2007, 92:541-560.

80. Simon C, Frati F, Beckenbach A, Crespi B, Liu H, Flook P: Evolution, weighting, and phylogenetic utility of mitochondrial gene sequences and a compilation of conserved polymerase chain reaction primers. Ann Entomol Soc Am 1994, 87:651-701.

81. Martins J, Solomon SE, Mikheyev AS, Mueller UG, Ortiz A, Bacci M: Nuclear mitochondrial-like sequences in ants: evidence from Atta cephalotes (Formicidae: Attini). Insect Mol Biol 2007, 16:777-784.

82. Behura SK: Analysis of nuclear copies of mitochondrial sequences in honeybee (Apis mellifera) genome. Mol Evol Biol 2007, 24:1492-1505. 
83. Pamilo P, Viljakainen L, Vihavainen A: Exceptionally High Density of NUMTs in the Honeybee Genome. Mol Biol Evol 2007, 24:1340-1346.

84. Wyman SK, Jansen RK, Boore JL: Automatic annotation of organellar genomes with DOGMA. Bioinformatics 2004, 20:3252-3255.

85. Laslett D, Canbäck B: ARWEN: a program to detect tRNA genes in metazoan mitochondrial nucleotide sequences. Bioinformatics 2008, 24:172-175.

86. Lowe TM, Eddy SR: tRNAscan-SE: a program for improved detection of transfer RNA genes in genomic sequence. Nucleic Acids Res 1997, 25:955-964.

87. Eddy SR, Durbin R: RNA sequence analysis using covariance models. Nucleic Acids Res 1994, 22:2079-2088.

88. Edgar RC: MUSCLE: multiple sequence alignment with high accuracy and high throughput. Nucleic Acids Res 2004, 32:1792-97.

89. Posada D: jModeltest: phylogenetic model averaging. Mol Biol Evol 2008 , 25:1253-1256.

90. Rozas J, Sánchez-DelBarrio JC, Messeguer X, Rozas R: DnaSP, DNA polymorphism analyses by the coalescent and other methods. Bioinformatics 2003, 19:2496-2497.

91. Morton BR: Chloroplast DNA codon use: Evidence for selection at the $p s b$ A locus based on tRNA availability. J Mol Evol 1993, 37:273-280.

92. Wright F: The "effective number of codons" used in a gene. Gene 1990, 87:23-29.

93. Shields DC, Sharp PM, Higgins DG, Wright F: "Silent"sites in Drosophila genes are not neutral: Evidence of selection among synonymous codons. Mol Biol Evol 1988, 5:704-716.

94. Yang Z: PAML 4: a program package for phylogenetic analysis by maximum likelihood. Mol Biol Evol 2007, 24:1586-1591.

95. Albu M, Min XJ, Hickey D, Golding B: Uncorrected nucleotide bias in mtDNA can mimic the effects of positive Darwinian selection. Mol Biol Evol 2008, 25:2521-2524.

96. Milne I, Lindner D, Bayer M, Husmeier D, McGuire G, Marshall DF, Wright F: TOPALi v2: a rich graphical interface for evolutionary analyses of multiple alignments on HPC clusters and multi-core desktops. Bioinformatics 2009, 25:126-127.

97. Martin DP, Williamson C, Posada D: RDP2: recombination detection and analysis from sequence alignments. Bioinformatics 2005, 21:260-262.

98. White DJ, Wolff JN, Pierson M, Gemmell NJ: Revealing the hidden complexities of mtDNA inheritance. Mol Ecol 2008, 17:4925-4942.

99. Huelsenbeck JP, Ronquist F: MRBAYES: Bayesian inference of phylogenetic trees. Bioinformatics 2001, 17:754-755.

100. Guindon S, Gascuel O: A simple, fast, and accurate algorithm to estimate large phylogenies by maximum likelihood. Syst Biol 2003, 52:696-704.

101. Anisimova M, Gascuel O: Approximate Likelihood-Ratio Test for Branches: A Fast, Accurate, and Powerful Alternative. Syst Biol 2006, 55:539-552.

102. Wong KM, Suchard MA, Huelsenbeck JP: Alignment uncertainty and genomic analysis. Science 2008, 319:473-476.

103. Martin D, Rybicki E: RDP: detection of recombination amongst aligned sequences. Bioinformatics 2000, 16:562-563.

104. Padidam M, Sawyer S, Fauquet CM: Possible emergence of new gemini viruses by frequent recombination. Virology 1999, 265:218-225.

105. Martin DP, Posada D, Crandall KA, Williamson C: A modified bootscan algorithm for automated identification of recombinant sequences and recombination breakpoints. AIDS Res Hum Retroviruses 2005, 21:98-102.

106. Maynard Smith J: Analyzing the mosaic structure of genes. $J \mathrm{Mol}$ Evol 1992, 34:126-129.

107. Posada D, Crandall KA: Evaluation of methods for detecting recombination from DNA sequences: Computer simulations. Proc Natl Acad Sci USA 2001, 98:13757-13762.

108. Gibbs MJ, Armstrong JS, Gibbs AJ: Sister-Scanning: a Monte Carlo procedure for assessing signals in recombinant sequences. Bioinformatics 2000, 16:573-582.

109. Boni MF, Posada D, Feldman MW: An exact nonparametric method for inferring mosaic structure in sequence triplets. Genetics 2007, 176:1035-1047.

110. Weiller GF: Phylogenetic profiles: a graphical method for detecting genetic recombinations in homologous sequences. Mol Biol Evol 1998, 15:326-335.

111. Holmes EC, Worobey M, Rambaut A: Phylogenetic evidence for recombination in dengue virus. Mol Biol Evol 1999, 16:405-409.
112. McGuire G, Wright F: TOPAL 2.0: Improved detection of mosaic sequences within multiple alignments. Bioinformatics 2000, 16:130-134.

113. Husmeier D, Wright F: Probabilistic Divergence Measures for Detecting Interspecies Recombination. Bioinformatics 2001, 17(Suppl 1):S123-S131.

114. Husmeier D, McGuire G: Detecting Recombination in 4-Taxa DNA Sequence Alignments with Bayesian Hidden Markov Models and Markov Chain Monte Carlo. Mol Biol Evol 2003, 20:315-337.

115. Sawyer SA: Statistical tests for detecting gene conversion. Mol Biol Evol $1989,6: 526-538$

doi:10.1186/1471-2148-10-300

Cite this article as: Gotzek et al:: Mitochondrial genome evolution in fire ants (Hymenoptera: Formicidae). BMC Evolutionary Biology 2010 10:300.

\section{Submit your next manuscript to BioMed Central and take full advantage of:}

- Convenient online submission

- Thorough peer review

- No space constraints or color figure charges

- Immediate publication on acceptance

- Inclusion in PubMed, CAS, Scopus and Google Scholar

- Research which is freely available for redistribution

Submit your manuscript at www.biomedcentral.com/submit
C Biomed Central 